(C) 2011 IEEE. Reprinted, with permission, from Priyadarsi Nanda, Credibility Problems and Tradeoff between Realistic and Abstraction in WANET and WSN Simulation . Wireless Communications, Networking and Mobile Computing (WiCOM), 2011 7th International Conference on, September 2011. This material is posted here with permission of the IEEE. Such permission of the IEEE does not in any way imply IEEE endorsement of any of the University of Technology, Sydney's products or services. Internal or personal use of this material is permitted.

However, permission to reprint/republish this material for advertising or promotional purposes or for creating new collective works for resale or redistribution must be obtained from the IEEE by writing to pubs-permissions@ieee.org. By choosing to view this document, you agree to all provisions of the copyright laws protecting it 


\title{
Credibility Problems and Tradeoff between Realistic and Abstraction in WANET and WSN Simulation
}

\author{
Dong Yu, Priyadarsi Nanda, Robin Braun \\ School of Computing and Communication \\ University of Technology, Sydney, \\ Australia \\ (10743387, Priyadarsi.Nanda, R.Braun)@uts.edu.au
}

\begin{abstract}
Wireless network Simulation is challenging due to the effect of the dynamic and fading channel. The task is even more challenging in Wireless Sensor Network (WSN), adding the factor of energy source limitation. Validating simulation results with real implementation are still an unresolved question in wireless research in general and in particular WSN. There are few standard procedures to follow which guarantee accuracy and credibility in terms of answering the question in hands. On one hand, simulation results are aimed towards as realistic as possible conforming to real world implementation. On the other hand, abstraction can eliminate the fragmentation of hardware prototypes, operating system models and different simulation tools. In our research we try to tackle credibility problem in WSN with tradeoffs between realistic and abstraction, and setup the principles and guideline for practical simulations in WSN.
\end{abstract}

Keywords-credibility; abstraction; realistic ; wireless; tradeoff

\section{INTRODUCTION}

After about a decade of research and development efforts worldwide, Wireless Sensor Network (WSN) research is still heavily fragmented. There is not yet any standard and technology compared to some other fields of networking research, those in fact have achieved a predominance status. At present, the market standards in WSN include ZigBee, EnOcean, 802.15.4 and various protocols at different layers. There are a lot of challenges for the WSN sector and the need for harmonization of research in the following areas.

Hardware platform: Above mentioned technology standards normally lead to different sensor node prototypes as hardware platforms. If a consolidation of one or two dominance prototypes could be achieved, the economies of scale would make such an effort worthwhile by making nodes available in large quantities at low cost like current PC desktop and laptop have experienced [1]. Although different prototypes are not problems as long as their idiosyncrasies can be easily abstracted away, this convergence to dominance hardware platform can still further benefit the hardware abstraction of WSN operating system.

Simulation environments: Although there are a plethora of simulation tools used in WSN research community for performance evaluation, like $\mathrm{Ns} / 2$, Opnet, OmNEt++,
Qualnet, GlomoSim, but none of these simulation tools perfectly meet the demands of WSNs. This is partly due to some simulation tools are originally designed for wired network; and even some simulation tools aim specifically at WSN performance evaluation, but the authors of the tools did not catch the complex essence of WSN at the time of simulation design.

The design of wireless sensor networks requires us to simultaneously consider the effects of several factors such as energy efficiency, fault tolerance, quality of service demands, synchronization, scheduling strategies, system topology, and communication and coordination protocols. The real world WSN is in a state of constant change. Individual node residual energy is changing in different pace according to different routing strategies and the position the node is in. The topology is changing more often than any other form of networks due to the dynamic nature of node state and wireless communication medium state. This uncertainty is common for WSN but rare in wired network, even in other form of wireless network where energy recharging is not problematic.

Reflecting the current available simulation tools [1]:

1. The wireless channel model is too simplistic and not easily changed (NS/2), 2. Not all relevant protocols are easily available (Omnet), 3. Some tools are commercial (Opnet, Qualnet) involving high cost, 4. Some tools are too infrequently used to easily allow comparison of results with work of other researchers (all tools with the possible exception of NS/2).More importantly, to our knowledge, there is no simulation tool for which convincing models of applications are available.

\section{CURRENT Simulation CREDiBiLity Status IN WIRELESS AD HOC AND SENSOR NETWORK RESEARCH COMMUNITY}

Kurkowski et al. [3] and Andel et al. [4] have highlighted the fact that a large portion of published papers in the area of wireless Ad Hoc and sensor network lacks the level of detail to allow for the precise independent replication of their proposed solutions. Normally the researchers just show the simulation resulting figures on the paper omitting the detailed 
simulation developing process, sometimes even not mentioning the simulation tools.

Yuri et al in [5] further pointed out that, the simulation problem is not only limited to insufficient information about the simulation platform and assumptions on the environment and traffic sources, but also there is frequently lack of sufficient details in the description of the protocol itself that leaves unintentional degrees of freedom in the implementation, which impact the resulting performance or even correctness of the proposed solution. Yuri et al. in [5] try to implement the T-MAC protocol in the Castalia simulator, They used their own experience in replication of the simulation process, concluded that even though T-MAC is a successful MAC protocol, however there are several points in its description that are left ambiguous, leading to several design choices. Design choices in a protocol are desirable only when they are deliberate and presented clearly to the implementers. Otherwise they can lead to different implementations with different performance characteristics.

Another WSN simulation problem is modeling difficulties which are far more serious and inherent in wireless sensor network with dynamic and constrained nature both in energy and channel environment. According to [11, 12, 13], simulators do not always reflect the performance of the modeled wireless sensor processes mainly due to inaccuracies in the physical model such as interferences, error models, and power consumption.

Watteyne concluded in [10] that a successful WSN simulator should have following properties or constraint requirements:

- Accurate propagation model supporting WSN to communicate in dynamic and time-changing channel.

- Proper power consumption model is necessary for all realistic application to tackle energy constraint problem.

- Model Extensibility with simulators supporting active research in the area of WSN. Simulator extensibility is a must for researchers to apply their novel idea and algorithms.

- High Scalability supporting wide range of sensors in application simulation without degradation in performance.

- Less complex usability and easy to access functionalities bringing the success of the simulator for long run.

- Less Cost bringing the tool to be popular among user and researchers and develop new applications for the future.

However, it is observed with most deployed WSN that complex radio and energy models can generate extensibility difficulty, inconvenience to use and further high cost of modeling. The authors in [7], who invented the famous WSN simulator Castalia, claim that Castalia has the most accurate wireless channel and radio models found in current literature. Based on the work of Zuniga et al. [15], detailed radio behaviour forces the user to deal with many of the unpleasant aspects of communication, which make it difficult to use. Castalia also features a flexible physical process model, takes into account usually neglected issues such as clock drift, sensor bias, sensor energy consumption, CPU energy consumption, and monitor of resources such as memory usage and CPU time.

On the basis of all the above mentioned modelling tools, it is not clear if they can accurately simulate and predict high level WSN protocol behaviour as in real deployments. To answer these questions, there have been investigations on how multi parameter tunable MAC protocol behaves in a real deployment, so as to take a first step towards validating and possibly tuning Castalia. Even though they created the same wireless channel conditions as in the real deployment, there is still significant disagreement between real deployment and simulation based results. The explanation of these disagreements is part of the ongoing work to validate the simulator and understand if there are some fundamental issues we are missing and should be modeled in the simulation [7].

Until now the results about the studied simulation environments are mainly based on papers, manuals, documentation and partly source code review. For that reason, the provided results are rather theoretical and might be different to practical experience. There is few research papers published based on real world WSN test-bed. In [6] Philipp et al studied WiseMAC, an energy-efficient MAC protocol for wireless sensor networks in simulation with OMNeT++ simulator and on a real sensor hardware test-bed: Embedded Sensor Boards (ESB), which is a sensor node platform along with its own operating system, ScatterWeb OS. They illustrated how simulation models can be verified by crosscomparing simulation results with real-world experiment results. The authors claimed that by careful calibration of simulation model parameters, the inevitable gap between simulation models and real-world conditions can be reduced. But such a scheme has following problems:

- It is not ubiquitous prompting: not every researcher can enjoy the luxury of test-bed experiment.

- In case of some novel research ideas being in their infancy, before researchers fully understand the details of the algorithm, a proof-of-concept with simulation must be deployed as a prelude to further speculation.

So we must provide a way at least to give a rough idea about the investigated algorithm, though not exactly as realistic as real world implementation, but not at least to mislead the direction of further study. 


\section{SimULATION WITH PURPOSE: THE LEVEL OF ABSTRACT AND CREDIBILITY TRADEOFF}

There is no de facto simulator supporting all types of applications in WSN simulation. Given the facts that any simulation is not perfect and that there are a number of popular sensor simulators available, different simulators are only appropriate and effective in certain aspects of simulation scenarios. It is important for developers to choose a simulation tool to suit their purpose.

Simulation is a process of abstraction. Before we elaborate further on this vein, let's first consider the purposes of simulation. Simulation is an alternative realization that approximates the system [17]. The purpose of simulation is to analyze and understand the system's behavior under various alternative actions or decisions. Normally the systems are large and complex, and would be difficult and expensive to experiment in real life. The key component of a simulation is the simulation model, which is simplified and abstract representation of the system. Due to simplicity in requirement, some details are intentionally omitted and the measurement in simulation will never exactly be the same as real world implementation. Obviously if researchers put more details in the simulation model, the simulation result may more mimic the reality, such a simulation is more realistic. But even if we do not account on the cost of the efforts researchers put on the detailed modeling and implementation, the problems of too detailed modeling still exist and can be represented through the following:

- Development time and simulation execution time can be more challenging and bugs are less likely to be detected once modeling details increases.

- Too many details can distract the research problem at hand, the effect of the main concerned parameters may be insignificantly merged in the mass details.

On the other hand, higher level abstraction can distill a research question to its essence and simulation provides insight not colored by arbitrary details of specific proposed solutions. A more abstract simulation can also make the effects of a change in algorithm distinct, where they would be obscured by other effects in a more detailed simulation.

Finally, omission of simulation details can improve performance by multiple orders of magnitude [18]. But omitting detail or oversimplifying the model can lead to ambiguous or erroneous outcomes. It is difficult for researcher to decide which details relate to the question under investigated. Is the wireless channel complexity the significant factor to the problem at hand and worthy detailed modeling? Advanced research topics usually accompany with some extent of uncertainty. With new protocols, it is even more difficult to estimate the effect of the abstraction undoubtedly.
Along with the definition of simulation in [17], to fulfill the task of understanding system behavior, authors in[16], further pointed out the factors causing unrealistic results in Wireless ad hoc and sensor networks, included but not limited to : inappropriate radio models, unrealistic application traffic, and lack of real world implementations. But Stojmenovic in [14] argues that we should not only consider system simulation as a way to mimic the real world behavior, and judge the success or failure of simulation. We should emphasis the proof of concept side of simulation. We further elaborate in this direction. If we know every aspects of the protocol, like in wired network some Internet protocols, simulation falls to the category of education tool; we can focus on more realistic part of the simulation purpose. But in wireless sensor and other hot research topics, new inventions, novel ideas need simulation to prove feasibility, compare alternative solutions and decide further research direction.

So Stojmenovic defined the purpose of simulation with proof of concept to make unknown known; a very simple model (high abstract) and scenario, matching model with assumptions used to design protocol. The purpose is to demonstrate that what is expected about the very basic performance is indeed true. Simulations are simply replacing theoretical proofs of performance because later are very difficult (often probably impossible) to derive, so that basic claims and expectations are confirmed. But Stojmenovic in his argument lightly ignored the skills requirement of high abstraction. In our view, it does not mean an easy task which brought more labor before simulation, in contrast with heavy parameters tweaking at implementation time as low abstraction.

Researcher must understand the assumptions' effects on the overall outcome. Not omitting the important factors involved. Otherwise, a misleading result will cause the whole protocol design fails in real implementation. Researcher must judge what level of detail is required for a given question. Wireless simulations raise many new questions about appropriate levels of detail in simulation models for radio propagation and energy consumption, which may or may not relevant to many areas of research in WSN. Our final thought in this argument is to choose a model to suit our purpose, like the decision on simulation tool.

\section{WSN SIMULATION PRACTICE GUIDELINE AND FUTURE STANDARDIZATION DIRECTION}

"Fully realistic" simulation is impossible in any simulation practice, even in mature technologies coupled with specific tailored simulation tool, like NS2 for stable and standardized Internet protocols. WSN research and industry are still in nascent state, far from its prosperity and widespread as experts predicted. However, WSN has showed its potentials to fit in pending pervasive computing and Internet of Things era. There are still a plethora of standards that causes confusion in 
the market and dispersion in the research efforts, which is adversary to healthy growth both in industry and research. Moreover these phenomenon cause difficulties in credible WSN simulation. This multitude of prototypes often renders experimental results difficult to compare; Radio modems have different modes of operations with different power consumptions, resulting in differently optimized lower-layer protocols. While these solutions are important and necessary as startup efforts, the next logic step is merging and compatibility towards more abstract standard, which is based on solid experiment results. Further research will base on the authentic model established both at analytical and simulation stages. Simulation results will be comparable.

In TinyOS, Researchers have put early efforts on abstraction of diverse hardware prototypes. They have foreseen hardware differences between mote platforms, namely Rene, Mica, and Mica2, affected software structure and networking capabilities in unpredictable way. So they developed networking abstraction mechanism called AM (Active Messages) used throughout TinyOS to alleviate effects of implementationspecific lower layers of the radio stack. These protocols embody very different assumptions about network stack composition and, as such, have limited interoperability. In principle, wireless sensor networks would benefit from a unifying abstraction. However, it has been suggested in [20] that, this abstraction should be close to the link level rather than the network level as TinyOS does. Because processing potentially occurs at each hop, not just at the end points, and there are many application-specific multipoint communication patterns (collection, aggregation, dissemination), nevertheless, they are excellent start in this direction.

Standardization cannot be solved by individual research group or company. Abstraction can be achieved by individual efforts. But standards have to be achieved by a consortium of industry and international organization.

\section{CONCLUSION}

In this paper we have tackled the simulation credibility problem in WSN. There is a lack of convincing simulation results to establish credible and dominant protocol suites. We foresee that future industry consolidation of abstraction and standardization will lead to simulation modeling based on solid foundation. But before such things become reality, we must carefully choose the right level of abstraction to effectively solve research problems involving WSN. Simulation purpose and difficulties are further clarified. In current exploring stage of WSN research, we argue that simulation should focus more on proof of concept to guide in right research direction to validate against real life implementations.

\section{References}

[1] Vlado Handziski, Andreas Köpke, Holger Karl,and Adam Wolisz. "A Common Wireless Sensor Network Architecture?". Sentzornetze, July 2003

[2] C. Mallanda, A. Suri, V. Kunchakarra, S. S. Iyengar, R. Kannan, A. Durresi, and S. Sastry. "Simulating wireless sensor networks with omnet++." 2005

[3] S. Kurkowski, T. Camp, and M. Colagrosso. "MANET Simulation Studies: The Incredibles", Mobile Computing and Communications Review 9(4): 56-61, October 2005.

[4] T.R Anel and A. Yasinsac. "On the credibility of MANET simulations", IEEE Computer 39(7):48-54, June 2006.

[5] T. Yuri, B. Athanassios and L. Lavy. "Experiences and Lessons from Implementing a Wireless Sensor Network MAC Protocol in the Castalia Simulator" submitted to IEEE Wireless Communications \& Networking Conference 2010 (WCNC 2010), Sydney, Australia.

[6] H. Philipp and B. Torsten. Calibrating Wireless Sensor Network Simulation Models with Real-World Experiments" NETWORKING 2009, LNCS 5550, pp. 1-13, 2009.

[7] H. Pham, D. Pediaditakis, and A. Boulis "From Simulation to Real Deployments in WSN and Back" in Proceedings of the 8th ACM International Symposium on a World of Wireless, Mobile and Multimedia Networks (WOWMOM '07), pp. 1-6, Helsinki, Finland, June 2007

[8] D. Kotz, C. Newport, R. Gray, J. Liu, Y. Yuan, and C. Elliott, "Experimental evaluation of wireless simulation assumptions," in Int'l Workshop Modeling Analysis and Simulation of Wireless and Mobile Systems (MSWiM04). ACM Press, New York, Oct. 2004

[9] K. Marko, S. Mikko, K. Niilo and T. Pirkka "Survey of Wireless Sensor Networks Simulation Tools for Demanding Applications" 2009 Fifth International Conference on Networking and Services.

[10] T. Watteyne. "Using Existing Network Simulators for Power-Aware Self-Organizing Wireless Sensor Network Protocols" http://arxiv.org/abs/cs/0611056

[11] David Cavin , Yoav Sasson , André Schiper, "On the accuracy of MANET simulators," Proceedings of the second ACM international workshop on Principles of mobile computing, October 30-31, 2002,

[12] F. Haq and T. Kunz. Simulation vs. emulation: evaluating mobile ad hoc network routing protocols. In Proceedings of the International Workshop on Wireless Ad-hoc Networks (IWWAN'05), May 2005.

[13] Victor Shnayder, Mark Hempstead, Borrong Chen, Geoff Werner Allen, and Matt Welsh. "Simulating the Power Consumption of LargeScale Sensor Network Applications." SenSys'04, November 2004, Baltimore, Maryland, USA.

[14] I. Stojmenovic, "Simulations in wireless sensor and Ad Hoc networks: Matching and advancing models, metrics, and solutions," IEEE Commun. Mag., vol. 46, no. 12, pp. 102-107, Dec. 2008.

[15] M. Zuniga, B. Krishnamachari, "Analyzing the Transitional Region in Low Power Wireless Links," First IEEE International Conference on Sensor and Ad hoc Communications and Networks (SECON), Santa Clara, CA, October 2004.

[16] Andel, T.R., and Yasinsac, A. "On the credibility of manet Simulations", IEEE Computer (July 2006), 48-54.

[17] A. Seila "Introduction to Simulation" Proceeding of the 1995 Winter Simulation Conference

[18] J. Heidemann, N. Bulusu, J. Elson, C. Intanagonwiwat, K. Lan, Y. Xu, W. Ye, D. Estrin, and R. Govindan. "Effects of detail in wireless network simulation." In Proceedings of the SCC Multiconference on Distributed Simulation,USA, January 2001.

[19] W. Tranter, K. Shanmugan, T. Rappaport, K. Kosbar "Principles of Communication Systems Simulation with Wireless Applications" PRENTICE HALL 2003

[20] D. Culler, P. Dutta, C. T. Ee, R. Fonseca, J. Hui, P. Levis, J. Polastre, S. Shenker, I. Stoica, G. Tolle, and J. Zhao. "Towards a sensor network architecture: Lowering the waistline." HotOS, 2005 